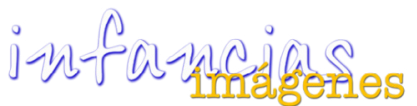

http://revistas.udistrital.edu.co/ojs/index.php/infancias

DOI: 10.14483/udistrital.jour.infimg.2016.2.a02

IMÁGENES DE INVESTIGACIÓN

\title{
El bilingüismo y su incidencia en el léxico infantil bilingüe*
}

\author{
Bilingualism and Itts Impact on the Bilingual Children's Lexicon \\ Martha Luz Valencia Castrillón ${ }^{1}$
}

Para citar este artículo: Valencia, M. L. (2016). El bilingüismo y su incidencia en el léxico infantil bilingüe. Infancias Imágenes, 15(2), 194-207.

\section{Resumen}

Esta investigación tiene como objetivo describir la relación entre el bilingüismo y la densidad y diversidad léxica en español y en inglés de sujetos en formación bilingüe (español-inglés). Se utilizó una metodología cional, comparativo y explicativo. La población está conformada por 66 niños entre ocho y nueve años de edad de tercer grado de primaria de dos instituciones educativas públicas del Quindío, Colombia. Se aplicaron dos tipos de instrumentos: de captura de información y de medición de variables. Se confirma que existe una relación de incidencia entre el bilingüismo, la densidad y diversidad léxica escrita en español y en inglés. Los resultados que arroja este estudio proporcionan información fundamental para investigadores, padres, profesores y administradores del sector educativo en su responsabilidad de ofrecer las mejores condiciones para el desarrollo apropiado del bilingüismo en el aula de clase.

Palabras clave: Educación bilingüe, escuela primaria, densidad léxica, diversidad léxica, sector educativo.

Recibido: 24-junio-2016 / Aprobado: 10-agosto-2016

\begin{abstract}
This research study has as the objective to describe the relationship between bilingualism and the lexical density and diversity in Spanish and English of subjects in bilingual formation (Spanish-English). A research methodology that is not experimental but relational, comparative and explicative was used. The population of this study is composed by 66 children between eight and nine years old, they are third graders of two public schools of Quindío, Colombia. Two types of instruments were used: capturing information and measuring variables. It was found that a relationship of incidence exists between bilingualism and lexical density and diversity in Spanish and English. The results give fundamental information for researchers, parents, teachers and administrators of the education field in their responsibility to offer the best conditions for the appropriate development of bilingualism in the classroom.
\end{abstract}

Keywords: Bilingual education, primary school, lexical density, lexical diversity, education field.

\footnotetext{
* Este artículo es resultado de la investigación titulada "Organización cognitiva, densidad y diversidad léxica, y producción descriptiva escrita bilingüe de sujetos en formación bilingüe de tercer grado de primaria"; de la Universidad del Quindío; fecha de inicio: 01-05-2014, fecha de terminación: 01-08-2016.

1 Licenciada en Lenguas Modernas. Magíster en Didáctica del Inglés. Estudiante del Doctorado en Ciencias de la Educación RUDECOLOMBIA. Docente, Universidad del Quindío. Correo electrónico: maluval28@gmail.com.
} 


\section{Introducción}

El siglo XXI y la globalización imponen al ser humano un reto lingüístico: el uso de una lengua adicional $(\mathrm{L} 2)^{2}$ a la materna $(\mathrm{LM})^{3}$, o sea, el bilingüismo. Desde el dominio social, cultural, lingüístico y educativo, entre otros, existen distintas razones que demuestran la importancia y, por lo tanto, la actualidad del bilingüismo en el mundo de hoy. El bilingüismo se ha convertido en un factor esencial para el progreso de las naciones, la expansión de la industrialización, las relaciones comerciales, los avances científicos y tecnológicos. En este sentido, Blanco (2007) sostiene que los nuevos rumbos que ha tomado el mundo en pro de la globalización convierten a los seres humanos en agentes necesitados del conocimiento que se adquiere de otras culturas por medio del lenguaje.

El bilingüismo hace referencia al contacto de lenguas en el sujeto o en el grupo social (Hamers y Blanc, 2000; Baker, 2006) al igual que a fenómenos de orden social relativos a la cotidianidad a la que se enfrenta el individuo, la familia, la comunidad y la sociedad en general, tales como: los matrimonios entre personas que no poseen la misma LM, la migración de tipo política y religiosa, la economía, el comercio, además de la educación y la cultura (Lewis, 1976; Grosjean, 2010). Se considera que muchas personas son bilingües, ya que deben enfrentar experiencias comunicativas y necesidades específicas con el uso de una o varias lenguas (Snow, 1999).

La presente investigación, de corte cuantitativo y diseño no experimental transversal, se enmarca epistemológicamente en los desarrollos de las ciencias sociales, cognitivas y del lenguaje, y el bilingüismo. Existe un reconocimiento de la necesidad que tiene la sociedad actual de formar usuarios de varias lenguas, es decir ciudadanos bilingües. En este estudio se asume el bilingüismo en relación con varios aspectos: primero, como fenómeno lingüístico de gran presencia y de creciente actualidad

\footnotetext{
2 La abreviatura L2 se utilizará a lo largo de este documento para hacer referencia, en general, a la(s) lenguas adquiridas después de la lengua materna (LM).

3 Hamers y Blanc definen la LM como: "el código (o códigos) lingüístico que corresponde a la primera experiencia lingüística del sujeto" $(373,2000)$.
}

en el planeta (Abdelilah-Bauer, 2007). Segundo, se trabaja con un bilingüismo temprano y sucesivo (McLaughlin, 1984; Sánchez-Casas, 1999), es decir, el bilingüismo que se lleva a cabo durante la niñez y en el cual se adquiere un sistema lingüístico después del otro, la LM y luego la L2. Tercero, se toma en cuenta que es bilingüismo igualitario (Hagège, 1996), ya que la relación entre la L1 y la L2 es de igualdad dado al estatus social positivo de cada una de las dos lenguas. Finalmente, la investigación toma en cuenta el bilingüismo con dos lenguas internacionales, que son aquellas lenguas que utilizan entre sí personas de diferente origen o nación, y son usadas en todos los continentes para facilitar los lazos y la comprensión internacional (Phillipson, 1992), en este caso específico las lenguas internacionales son el español y el inglés.

\section{Las variables en estudio}

La organización cognitiva bilingüe: primera variable en estudio

La organización cognitiva bilingüe explora la relación pensamiento y lenguaje estudiando cómo se organiza y representa en el cerebro del sujeto bilingüe la información lingüística correspondiente a las lenguas que usa. Los estudios de la mente bilingüe se consolidan a comienzos de la década de 1950, al tratar la relación signo y significado en la organización mental de las lenguas del sujeto bilingüe con la tipología de bilingüismo coordinado, compuesto (o amalgamado) y subordinado, propuesta por Weinreich (1953). Este autor distingue tres situaciones relacionadas con los contextos de adquisición en contexto natural y/o escolar de las lenguas: 1) el sujeto bilingüe que mantiene sus dos sistemas lingüísticos separados en su representación lexical y en su representación conceptual: bilingüe coordinado; 2) el sujeto bilingüe que hace corresponder las representaciones lexicales en L1 y en L2 con una sola representación conceptual: bilingüe compuesto, cuyas hipótesis de funcionamiento de L2 se basan en gran medida en el funcionamiento de su $L 1 ; 3$ ) el sujeto bilingüe que accede a la representación lexical de la L2 a través de la representación lexical y conceptual de la L1 mediante la traducción: bilingüe subordinado. 
Las investigaciones con respecto a la forma en la cual los bilingües procesan la información lingüística, en lo relativo a la idea de uno o dos almacenes léxicos, tiene también una evolución cronológica a partir de la propuesta de Ervin y Osgood (1954), quienes hacen una distinción entre los bilingües compuestos y los coordinados, con la creencia de que la historia de adquisición y el contexto de aprendizaje de ambos idiomas era lo que determinaba la estrategia de procesamiento de la información. Los bilingües compuestos utilizaban un único almacén para el procesamiento, recurrían más a la traducción y por lo tanto presentaban un mayor número de interferencias. Los bilingües coordinados poseían estructuras neurológicas separadas subyacentes a cada uno de los idiomas, es decir, dos almacenes para procesar la información y, en consecuencia, tenían menos interferencias (Lambert, Havelka y Crosby, 1958).

\section{La densidad y diversidad léxica: segunda variable en estudio}

196 La densidad y diversidad léxica se evidencian al inicio de la etapa escolar cuando el niño empieza a emplear un nuevo léxico relacionado con las áreas curriculares y con nuevas formas gramaticales. A medida que avanza la escolaridad y la edad del niño, este se enfrenta a nuevos retos y tareas comunicativas en su LM y, en algunos casos debido a las nuevas tendencias de la educación bilingüe y de procesos de desarrollo bilingüe en los contextos escolares, también en una L2.

La densidad es la relación de palabras de contenido (PC) —-sustantivos, verbos, adjetivos y adverbios- y el número total de palabras en un texto (PP) (Johansson, 2008). Es decir, es la razón de las palabras con contenido semántico y el número total de palabras en la composición (Laufer, 1991), está fundamentada en el Type/token ratio (TTR), que es una medida de la variación del vocabulario de un texto oral o escrito, la cual se considera muy útil para monitorear cambios y variedad en el vocabulario. El número de palabras de un texto se denomina tokens, algunos de los cuales pueden estar repetidos; las palabras que no están repetidas son Ilamadas types. La relación entre el número de types y el número de tokens se conoce como type/ token ratio (Biber, Conrad y Leech, 2002).
Por otro lado, la diversidad léxica se refiere al número total de palabras diferentes: PC — sustantivos, verbos, adjetivos y adverbios-y de palabras funcionales (PF) -artículos, preposiciones, pronombres, conjunciones- (Heilmann, Miller, Nockerts y Dunaway, 2010), en comparación con el número total de palabras producidas en un texto (PP). En los textos producidos por los sujetos de la muestra en esta investigación, la diversidad léxica será una estimación eficiente e informativa del análisis de los tipos léxicos o palabras diferentes, en relación con el número total de palabras producidas en una tarea comunicativa. La diversidad léxica ha sido utilizada en numerosos estudios para estimar el tamaño del vocabulario expresivo en individuos de diferentes edades, también ha sido considerada como una medida robusta y sensible al desarrollo para medir habilidades léxicas de los niños en una tarea comunicativa (Swanson et al., 2005; Uccelli y Páez, 2007).

\section{Metodología y diseño de la investigación} Luego de haber planteado el problema de investigación y los aspectos relacionados con las variables del estudio, se procede a describir la metodología que se llevó a cabo durante el proceso investigativo.

Objetivos, pregunta e hipótesis de investigación A partir de las ideas que se desarrollaron en el planteamiento del problema surgen los objetivos, la pregunta y las hipótesis de investigación que direccionan el proceso investigativo.

\section{Objetivos de investigación}

\section{- Objetivo general}

Explicar la relación entre la organización cognitiva bilingüe y la densidad y diversidad léxica, en inglés y en español, de estudiantes en formación bilingüe (español-inglés) en tercer grado de primaria de dos instituciones públicas del Quindío.

\section{- Objetivos específicos}

- Diagnosticar a los sujetos de la muestra según su organización cognitiva bilingüe: coordinado/ compuesto/ subordinado (Weinreich, 1953; Ervin y Osgood, 1954) a partir de la identificación de las marcas interlinguales e intralinguales en los textos descriptivos escritos en L2. 
- Determinar los desempeños de los sujetos en formación bilingüe de la muestra en la densidad y diversidad léxica en inglés y en español.

- Comparar los desempeños de los sujetos según su tipología bilingüe en la densidad y diversidad léxica escrita en inglés y en español.

- Determinar la relación entre la organización cognitiva bilingüe y la densidad y diversidad léxica en inglés y en español de los sujetos de la muestra.

\section{Pregunta de investigación}

¿Cuál es la relación entre la organización cognitiva bilingüe y la densidad y diversidad léxica escrita, en inglés y en español, de estudiantes en formación bilingüe (español-inglés) de tercer grado de primaria de dos instituciones públicas del Quindío?

\section{Hipótesis de la investigación}

\section{Hipótesis general}

- H1 Existe relación entre la organización cognitiva bilingüe y la densidad y diversidad léxica escrita, en inglés y en español, de estudiantes en formación bilingüe (español-inglés) de tercer grado de primaria.

- HO No existe relación entre la organización cognitiva bilingüe la densidad y diversidad léxica escrita, en inglés y en español, de estudiantes en formación bilingüe (español-inglés) de tercer grado de primaria.

\section{Hipótesis de trabajo}

- H2 Los sujetos en formación bilingüe de tercer grado de primaria, cuya tipología bilingüe es coordinada, alcanzan un mejor desempeño en la densidad y diversidad léxica en inglés y en español que los sujetos compuestos y subordinados.

- HO Los sujetos en formación bilingüe de tercer grado de primaria, cuya tipología bilingüe es coordinada, no alcanzan un mejor desempeño en la densidad y diversidad léxica en inglés y en español que los sujetos compuestos y subordinados.

Enfoque, alcance y diseño de investigación Esta investigación se lleva a cabo desde el campo de la psicolingüística, la cual es una ciencia de carácter experimental, este estudio, se ubicó en el enfoque empírico-analítico, el cual, según Latorre, del Rincón y Arnal (1997), tiende a centrarse más en los aspectos cuantificables de los fenómenos educativos para constatar relaciones y explicaciones causales generalizables. Se pretendió hacer un énfasis en la descripción e interpretación de los datos recolectados por medio de la aplicación de varios instrumentos y diversos análisis estadísticos.

La presente investigación fue experimental no controlada debido a que los sujetos de la muestra se encuentran en su contexto natural y no hubo manipulación de variables (Hernández et al., 2010). Desde el inicio, los aspectos éticos fueron tenidos en cuenta, principalmente por tratarse de un estudio con niños. Se solicitó el consentimiento informado de los rectores, directores de grupo, profesores de las cátedras de inglés y de español y de los padres de familia o acudientes de los estudiantes para entrar a las aulas y realizar las actividades relacionadas con el proyecto como la aplicación de la ficha de información general del estudiante, la ficha de información general del grupo y recoger las producciones escritas en español y en inglés. La participación en el estudio fue voluntaria, se garantizó el carácter confidencial en relación a la identidad de sus participantes y de las instituciones educativas involucradas.

\section{La muestra}

Se trató de una muestra de tipo intencional no probabilística, formada por un grupo de 66 estudiantes, entre los ocho y los nueve años de edad, en desarrollo de un bilingüismo (español-inglés) individual, temprano, consecutivo y escolar de tercer grado de primaria. Con el fin de realizar la equivalencia de los sujetos de la muestra y asegurar que los participantes se presentaban al estudio en las mismas condiciones experimentales, se aplicó una ficha de información general de cada sujeto. Se seleccionaron los participantes con base en los siguientes requerimientos: 1) niños entre ocho y nueve años de edad; 2) niños que hubieran cursado al menos cuatro años en cada institución educativa escogida (jardín, transición, primero, segundo y actualmente tercer grado); 3) 
niños que no tuvieran acceso a clases extracurriculares de la L2; 4) niños que no tuvieran oportunidad de práctica de la L2 durante viajes a otros países o con personas de su núcleo familiar que tengan conocimiento de la L2 y la usen en el entorno familiar.

Para obtener la muestra de estudiantes en esta investigación se seleccionaron dos instituciones educativas públicas del Quindío, Colombia, pertenecientes al pilotaje del Ministerio de Educación Nacional en su programa Colombia Bilingüe. En la institución educativa 1 se implementa un sistema de inmersión en L2 (cinco horas de curso de lengua extranjera + ocho horas utilizando el inglés como lengua vehicular para la enseñanza de otras asignaturas); en la institución educativa 2, tienen el sistema de intensificación en L2 (tres horas de curso de lengua extranjera + cuatro horas utilizando el inglés como lengua vehicular para la enseñanza de otras asignaturas). Los niños de la muestra en este estudio han cursado en cada una de las instituciones educativas desde el nivel de transición hasta el grado tercero de primaria, que es el escogido para esta muestra, son cinco años en total en proceso de formación bilingüe. La L2 se desarrolla por medio de una intervención pedagógica en un contexto formal de aprendizaje.

Técnicas e instrumentos de recolección de datos y análisis estadístico

Se utilizaron técnicas cuantitativas y cualitativas para la recolección de datos. Para el diagnóstico de la organización cognitiva bilingüe se empleó una plantilla para detectar las marcas interlinguales e intralinguales. Para conocer los desempeños en la densidad y diversidad léxica en inglés y en español se aplicaron las formulas aritméticas seleccionadas para ambos casos y se usaron plantillas de conteo de palabras. El análisis de los datos se apoyó en una variedad de procedimientos estadísticos descriptivos e inferenciales.

Instrumentos de recolección de datos

Para la recolección de la información, se manejaron dos tipos de instrumentos: de captura de información y de medición de variables.

\section{- Instrumentos de captura de información}

La captura de información se llevó a cabo mediante la aplicación de tres instrumentos:

La ficha general del estudiante: se aplicó para conocer la información y antecedentes lingüísticos de cada participante, con el fin de establecer las equivalencias entre los sujetos y descartar aquellos que tuvieran ventajas en la L2 sobre la totalidad de los participantes debido a su formación complementaria en la L2, al contacto intercultural en la L2 o por miembros de su núcleo familiar que pudieran interactuar con ellos usando la L2.

La ficha general de cada grupo: se aplicó a los directores de curso para indagar acerca de las generalidades de los grupos con respecto a la intensidad horaria del inglés como cátedra y como lengua vehicular, las asignaturas que cursan y sobre las competencias que tienen en la producción escrita en inglés y en español.

Prueba de escritura de un texto descriptivo escrito en español y otro en inglés: se aplicó a cada sujeto con el fin de obtener la información para diagnosticar la organización cognitiva bilingüe y determinar la densidad y diversidad léxica escrita en inglés y en español.

\section{- Instrumentos de medición de variables}

La medición de las variables se llevó a cabo mediante la aplicación de dos instrumentos:

Plantilla de identificación de marcas interlinguales e intralinguales: para identificar en los textos descriptivos escritos en L2 las marcas o fenómenos lingüísticos (lexicales o sintáctico-lexicales) como indicadores para diagnosticar la tipología bilingüe de los sujetos de la muestra.

Plantillas para la densidad y diversidad léxica: diseñadas para plasmar los resultados del conteo del léxico y de la aplicación de la formula aritmética en cada uno de los textos descriptivos escritos en inglés y en español.

\section{Estudios preliminares}

Se realizó una prueba piloto con una muestra de 16 estudiantes. Se administraron todos los instrumentos con el fin de probar su funcionamiento y eficacia para la recolección de los datos requeridos. Los estudios preliminares — prueba piloto- que 
se llevaron a cabo en esta investigación permitieron verificar que los instrumentos adaptados o diseñados fueran confiables, válidos y, por lo tanto, adecuados para ser aplicados a la muestra total y definitiva.

\section{Interpretación de resultados}

\section{Resultados de la organización cognitiva bilingüe}

Para esta variable se propuso un análisis multivariado a partir de dos índices: las marcas interlinguales y las marcas intralinguales detectadas en el texto descriptivo escrito en L2 de los sujetos de la muestra por medio de la plantilla diseñada para este fin. Se hizo un conteo de cada una de las marcas y posteriormente se ponderaron. Las marcas interlinguales se ponderaron con un valor de dos y las intralinguales con un valor de cuatro. Se dio menor valor ponderado (VP) a las marcas interlinguales, considerando que el sujeto en formación bilingüe de la muestra estaría en una etapa inicial de aprendizaje de la L2. Por otro lado, se dio mayor VP a las marcas intralinguales, considerando que el sujeto en formación bilingüe de la muestra estaría en proceso de construcción de un bilingüismo coordinado, lo que requiere de un mayor esfuerzo cognitivo.

Con los valores totales (VT) de cada sujeto en las marcas interlinguales e intralinguales se realizó un análisis Cluster que, a partir del cómputo de una matriz de similitudes entre los sujetos, produce un dendograma que muestra las fusiones sucesivas de los individuos y finaliza exponiendo la forma en la cual los individuos quedan agrupados. Del análisis Cluster realizado con las variables (marcas interlinguales e intralinguales) se formaron tres grupos, a los cuales se les denominó G1 coordinado, G2 compuesto y G3 subordinado. Los grupos se conformaron con base en las características similares entre los sujetos y en las categorías para la organización cognitiva propuestas por Weinreich (1953) y Ervin y Osgood (1954).

La tabla 1 muestra la distribución de los grupos según la organización cognitiva bilingüe, el número de sujetos en cada grupo y el porcentaje de acuerdo a la muestra total de 66 participantes.

Tabla 1. Distribución de los sujetos según su organización cognitiva bilingüe.

\begin{tabular}{cccc}
\hline Grupo & $\begin{array}{c}\text { Organización cognitiva } \\
\text { bilingüe }\end{array}$ & Sujetos & Porcentaje \\
\hline G1 & Coordinado & 25 & $37,87 \%$ \\
G2 & Compuesto & 24 & $36,38 \%$ \\
G3 & Subordinado & 17 & $25,75 \%$ \\
\hline
\end{tabular}

Fuente: elaboración propia.

Organización cognitiva bilingüe-marcas interlinguales

Se realizó el análisis estadístico tomando los VT de cada sujeto que conforma el G1, G2 y G3 en las marcas interlinguales. La tabla 2 muestra estadísticas descriptivas en este aspecto para la totalidad del grupo y para cada grupo según su organización cognitiva bilingue.

El mayor promedio en las marcas interlinguales se da en el grupo G3 subordinado 7,76, siendo este grupo el que presenta la mayor homogeneidad (17,93\%) en el coeficiente de variación. El G1 coordinado y el G2 compuesto presentan resultados similares tanto en los promedios 3,83 y 3,12 como en la variabilidad $45,9416 \%$ y $45,6285 \%$. La prueba de hipótesis involucrada en el análisis de varianza arrojó un p-valor $=0,0001$, con un valor

Tabla 2. Resumen estadístico para las Marcas interlinguales en la totalidad del grupo y en cada grupo según su organización cognitiva bilingüe.

\begin{tabular}{ccccccc}
\hline Grupo & $\begin{array}{c}\text { Número de } \\
\text { sujetos }\end{array}$ & Promedio & Máximo & Mínimo & $\begin{array}{c}\text { Desviación } \\
\text { estándar }\end{array}$ & $\begin{array}{c}\text { Coeficiente de } \\
\text { variación }\end{array}$ \\
\hline G1 & 25 & 3,12 & 2,0 & 6,0 & 1,42 & $45,62 \%$ \\
G2 & 24 & 3,83 & 2,0 & 6,0 & 1,76 & $45,94 \%$ \\
G3 & 17 & 7,76 & 6,0 & 10,0 & 1,39 & $17,94 \%$ \\
Total & 66 & 4,57 & 2,0 & 10,0 & 2,44 & $53,54 \%$ \\
\hline
\end{tabular}

Fuente: elaboracion propia. 
de significación 0,05, a partir de lo cual se puede afirmar que existe diferencia estadísticamente significativa en el número de marcas interlinguales entre los grupos G1, G2 y G3.

La prueba de comparaciones múltiples entre los grupos mostró que existe diferencia en el número de marcas interlinguales entre el grupo G3 subordinado con relación al G1 coordinado y al G2 compuesto. Entre los grupos G1 y G2 se advierten resultados similares en estas marcas y, por tanto, no existe diferencia estadísticamente significativa. En la figura 1 se muestran estos resultados

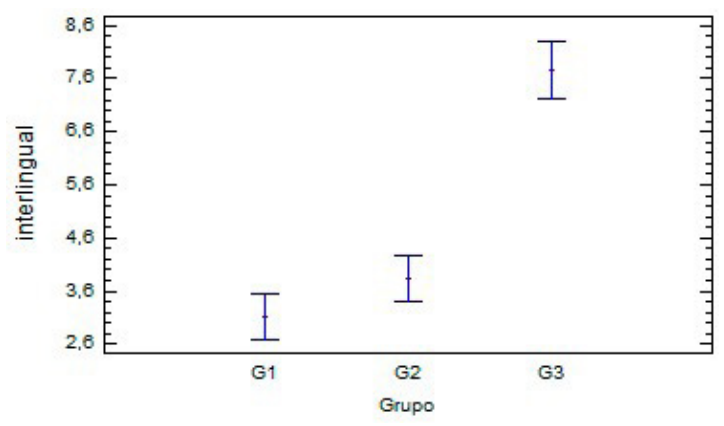

Figura 1. Promedio de marcas interlinguales en los tres grupos según la organización cognitiva bilingüe. Fuente: elaboración propia.

Organización cognitiva bilingüe-marcas interlinguales

Se realizó el análisis estadístico tomando los VT de cada sujeto que conforma el G1, G2 y G3 en las marcas intralinguales. La tabla 3 muestra estadísticas descriptivas en este aspecto para la totalidad del grupo y para cada grupo según su organización cognitiva bilingüe.

El mayor promedio de las marcas intralinguales se obtienen en el grupo G1 coordinado 9,60, siendo este grupo el que presenta la mayor homogeneidad en el coeficiente de variación (26,89\%). Se observa diferencia en los promedios de las marcas intralinguales entre los grupos G1 compuesto 3,33 y G3 subordinado 1,17. Los resultados de la prueba de hipótesis, con respecto a la comparación entre los tres grupos en el número de marcas intralinguales, mostró que existe diferencia significativa en el comportamiento del número de marcas intralinguales entre los grupos con $p$-valor $=0,0001$. Una prueba de comparaciones múltiples mostró que existe diferencia estadísticamente significativa entre cada uno de los grupos, con relación a las marcas intralinguales. La figura 2 muestra la representación de estos resultados.

Tabla 3. Resumen estadístico para las marcas intralinguales en la totalidad del grupo y en cada grupo según su organización cognitiva bilingüe.

\begin{tabular}{ccccc}
\hline Grupo & $\begin{array}{c}\text { Número de } \\
\text { sujetos }\end{array}$ & Promedio & $\begin{array}{c}\text { Desviación } \\
\text { estándar }\end{array}$ & $\begin{array}{c}\text { Coeficiente de } \\
\text { variación }\end{array}$ \\
\hline G1 & 25 & 9,60 & 2,58 & $26,89 \%$ \\
G2 & 24 & 3,33 & 1,52 & $45,68 \%$ \\
G3 & 17 & 1,17 & 1,87 & $159,68 \%$ \\
Total & 66 & 5,15 & 4,13 & $80,30 \%$ \\
\hline
\end{tabular}

Fuente: elaboracion propia.

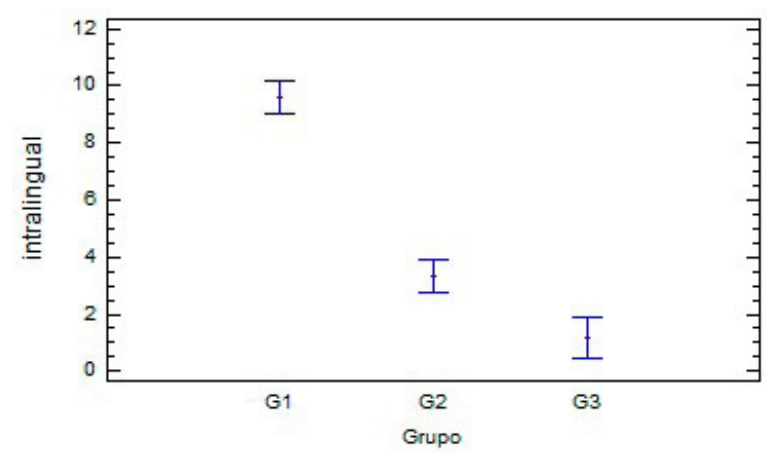

Figura 2. Promedio de las marcas intralinguales en los tres grupos según la organización cognitiva bilingüe. Fuente: elaboración propia.

El mayor promedio en las marcas interlinguales lo tiene el G3 subordinado 7,76, seguido del G2 compuesto 3,83 y del G1 coordinado 3,12. Por el contrario, el mayor promedio en las marcas intralinguales lo tiene el G1 coordinado 9,60, seguido del G2 compuesto 3,33 y del G3 subordinado 1,17. En la totalidad del grupo tienen mayor promedio las marcas intralinguales.

Un número mayor en el promedio de las marcas interlinguales por parte del G3 subordinado $(7,76)$ indicaría que el conocimiento limitado que estos sujetos tiene de la L2 los Ilevaría a acceder al significado de las palabras en esa lengua a través de la ruta lexical (Kroll, 1993). Así lo muestran las marcas prevalentes detectadas en esta investigación: traducción literal, interferencia, transferencia. La 
cantidad cercana de marcas interlinguales 3,83 e intralinguales 3,33 en el G2 compuesto sería evidencia de que poseen un conocimiento un poco más avanzado de la L2 que los llevaría a intentar el uso de la ruta conceptual (Potter et al., 1984) para tener acceso al significado de las palabras de la L2. Por ello, estos sujetos en ocasiones emplean formas inapropiadas de la L2 como algunas de las detectadas en este estudio: invención de palabras, generalización de reglas, formulación de hipótesis falsas. En el G1 coordinado el promedio de las marcas interlinguales es el más bajo de los tres grupos $(3,12)$, algunos de los sujetos de este grupo no presentan marcas interlinguales y otros empiezan a disminuir el número de las marcas intralinguales, lo anterior, se asocia a un mejor desempeño en su competencia en la L2 y a que en su interlengua las dos lenguas que usa tienden a irse separando. En este punto es donde según Signoret y Da Silva (2011), empieza el proceso de construcción de un verdadero bilingüismo.

Luego de diagnosticar a los sujetos de la muestra, según su organización cognitiva bilingüe, se procedió a dar respuesta al segundo objetivo de esta investigación que consiste en determinar la densidad y diversidad léxica en L1 y L2 de los sujetos en formación bilingüe de tercer grado de primaria. Estos resultados se obtuvieron por medio del conteo de palabras en los textos descriptivos escritos en L1 y L2 y la posterior aplicación de fórmulas aritméticas para cada aspecto.

Resultados de la densidad y diversidad léxica en inglés y en español

La clasificación de los sujetos de la muestra en los diferentes tipos de bilingües G1 coordinado, G2 compuesto y G3 subordinado según su organización cognitiva, permitió identificar las características de los niños en formación bilingüe y determinar su desempeño en la densidad y diversidad léxica en inglés y en español. Los rasgos cognitivos de los sujetos se reflejaron en la diferencia de los promedios de PF, PC del total de PP en ambas lenguas. Grosjean (2010) expresa que la mayoría de los bilingües usan sus lenguas para propósitos, situaciones y personas diferentes, no necesitan ser igual de competentes en las dos lenguas y el nivel de experticia que posean en cada habilidad de las lenguas depende del uso y los objetivos de comunicación.

Para el análisis de la densidad léxica se realizó el conteo del léxico de cada texto descriptivo escrito tanto en inglés como en español de los sujetos de la muestra. Se contó el número total de PP y el número total de PC (sustantivos, verbos, adjetivos y adverbios) y se aplicó la formula aritmética para obtener los valores definitivos de cada sujeto. Para la diversidad léxica, se realizó el conteo en los textos en ambas lenguas del número total de PP, y el número total de palabras diferentes de la siguiente manera: 1) El número total de $\mathrm{PC}$; 2) El número total de PF (artículos, preposiciones, pronombres, conjunciones). Se aplicó la fórmula para calcular la diversidad léxica.

Los textos descriptivos escritos en inglés y en español, como la tarea comunicativa seleccionada para analizar los desempeños de los sujetos en la densidad y diversidad léxica en inglés y en español, se diferencian ampliamente en su extensión. Lo anterior no es determinante para que los textos sean más o menos densos lexicalmente. Auza y Chávez (2013), han Ilamado la atención sobre la tarea comunicativa que se seleccione como muestra para analizar ya que esta influye en la producción léxica: entre más palabras produce el niño, menores oportunidades tiene de producir palabras diferentes. Es por esto que, aunque a simple vista y por obvias razones, los textos escritos en español sobrepasaron la extensión de los textos escritos en inglés; ambos mostraron un desempeño importante de los sujetos en su totalidad y por grupos según su organización cognitiva bilingüe. La misma lengua, o lenguas que se aprenden, se convierten en el recurso fundamental a través de los textos orales y escritos que los niños elaboran con diferentes intenciones y como respuesta a diferentes situaciones comunicativas. El léxico se hace accesible en su enorme diversidad con el fin de ser manipulado según la necesidad y las posibilidades de cada individuo.

A continuación, el diagrama de barras con la información de los promedios en la densidad léxica en inglés, en las PC y en las PP de los sujetos según su organización cognitiva bilingüe. 


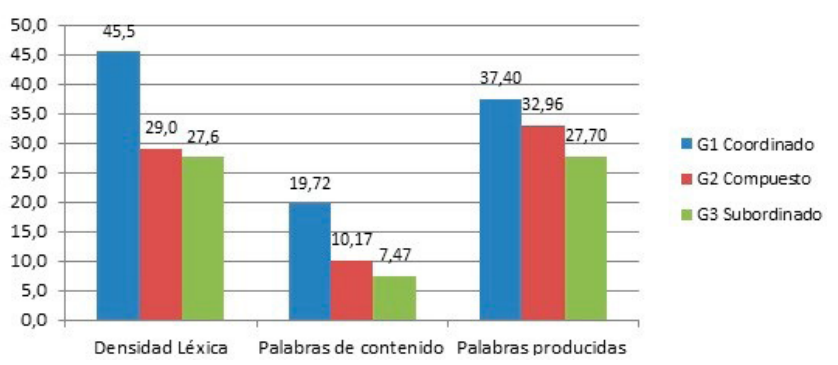

Figura 3. Densidad léxica en inglés, promedio de PC y de PP. Fuente: elaboración propia.

La densidad léxica en inglés mostró diferencia entre los tres grupos. El G1 obtiene el mayor promedio 45,5, con una amplia diferencia sobre el G2 con 29,0 y el G3 con 27,6. Con respecto a las PC, sigue existiendo una diferencia considerable entre el G1 y los otros dos grupos; el G1 tiene un promedio de 19,72, seguido del G2 con 10,17, y el G3 de 7,47 . Finalmente, en el promedio de PP, los promedios se acercan un poco: el G3 tiene el menor promedio con 27,70 seguido del G2 compuesto con 32.96 y del G1 coordinado con 37.40. En esta investigación, los resultados de la densidad léxica en inglés en el G2 compuesto y sobretodo en el G3 subordinado corroboran el hallazgo de Murillo (2009) en su estudio del léxico en niños costarricenses: las imprecisiones léxicas también dan cuenta de aquel vocabulario que está en proceso de apropiación semántica, pues el niño todavía no es capaz de abstraer y generalizar algunos rasgos semánticos que le permitan relacionar un significado específico con el significado que le corresponde. La presencia de términos imprecisos, inexistentes o equivocados encontrados en los textos descriptivos escritos en inglés por los niños de esta muestra constituye una señal que indicaría la etapa en la cual se encuentra cada niño en el proceso de adquisición de la L2 según su organización cognitiva bilingüe.

A continuación, el diagrama de barras con la información de los promedios en la densidad léxica en español, en las PC y en las PP de los sujetos según su organización cognitiva bilingüe.

Contrario a los promedios en la densidad léxica en inglés que mostraron amplia diferencia entre los tres grupos, especialmente, entre el G1 y los otros dos grupos G2 y G3, en la densidad léxica en español al parecer por ser la L1 de los sujetos, los 3 grupos obtienen promedios similares en todos los aspectos. El G1, 35,43, seguido del G2 con 33,56 y del G3 con 28,7. Las PC tiene como promedio en el G1 41,40, en el G2, 36,17 y en el G3, 24,71. La extensión de los textos en el G1 y G2 fue similar, con un promedio de PP de 108,44 y 105 sucesivamente.

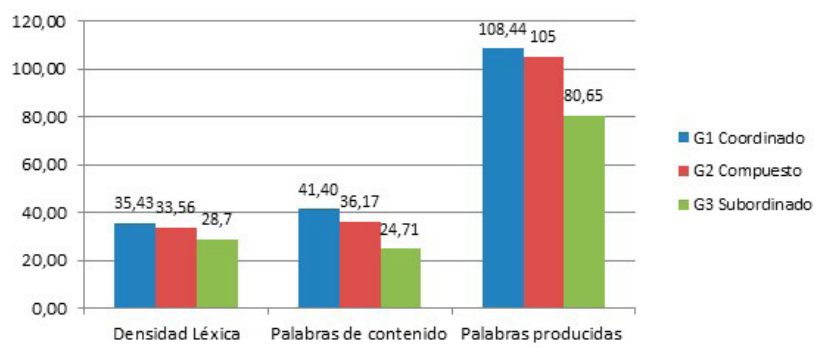

Figura 4. Densidad léxica en español, promedio de PC y de PP. Fuente: elaboración propia.

Por obvias razones, el desarrollo del léxico en la LM, en este caso el español, se hace más asequible a todos los sujetos de la muestra, razón por la cual los sujetos del G1, G2 y G3 tuvieron promedios similares en la densidad léxica, promedio de PC y promedio de PP en los textos descriptivos escritos en español. Múltiples investigaciones han puesto de relieve la importancia del input lingüístico en la adquisición de este aspecto del lenguaje. Se ha demostrado que una mayor riqueza del vocabulario se debe a una mayor exposición del niño a un input que tenga efectos importantes en el crecimiento de su léxico. Las situaciones que se plantean al niño para el desarrollo de su léxico, como en este estudio, la escritura de un texto descriptivo, son de capital importancia para la adquisición del lenguaje porque hace posible que el niño entienda qué hay detrás de unas palabras o de unas frases y ello le va a permitir construir los significados de estas (Aguado, 38, 1995).

La ventaja que muestran los sujetos de la muestra en la extensión del texto en español y del léxico utilizado en ellos se podría deber a que el desarroIlo funcional del lenguaje en la L1 se anticipa al estructural, por tanto, las funciones comunicativas que el lenguaje va a cumplir en años posteriores empiezan desde los primeros meses y se constituyen en los andamiajes necesarios para la adquisición del lenguaje del niño en su etapa escolar. Por lo anterior, aunque los niños de esta investigación 
estén en desarrollo de un bilingüismo temprano por estar entre los ocho y nueve años y hayan iniciado este proceso en la etapa preescolar, tienen un recorrido mayor en el tiempo de exposición y de aprendizaje de su L1 que en el de su L2.

A continuación, el diagrama de barras con la información de los promedios en la diversidad léxica en inglés, en las PF, en las PC y en las PP de los sujetos según su organización cognitiva bilingüe.

Los promedios del G1 son: en la diversidad léxica 0,63 , en las PF 6,60, en las PC 16,92 y en las PP 37,40. Este grupo obtiene los mejores resultados, seguido del G2 compuesto y del G3 subordinado que tienen promedios más bajos cercanos entre sí. Entre el G2 y el G3 se encuentra ubicado el $62 \%$ de muestra total de esta investigación. Rojas (2005), en su estudio del léxico en textos escritos por escolares de cuarto y sexto grado, encontró un desempeño similar al de los sujetos de este estudio: más de la mitad de los textos analizados resultaron con bajo promedio en el léxico utilizado. Al respecto, el mismo autor se refiere a que es muy probable que la pobreza léxica encontrada en el estudiantado afecte la capacidad potencial para referirse al mundo, para mostrar sus relaciones y para captar abstracciones y que limite los aprendizajes de las diversas asignaturas del currículo escolar y manifiesten otras carencias en relación con lo conceptual. Lo anterior podría estar ocurriendo en el G3 y evidenciarse a través del uso tan limitado del léxico que se observa con el menor promedio en todos los aspectos: las PF 3,59, en las PC, 7,47 y en las PP 27,47.

A continuación, el diagrama de barras con la información de los promedios en la diversidad léxica en español, en las PF, en las PC y en las PP de los sujetos según su organización cognitiva bilingüe.

Se observa un resultado bajo en los promedios en la diversidad léxica en español en los tres grupos: G1 0,50, G2 0,44 y G3 0,32. La razón podría ser que al tener un promedio tan alto de PP G1 117,36, G2 101,54 y G3 74,06, las palabras se repitan con mucha frecuencia. Los textos más extensos, en este caso los escritos en español, no son siempre los que presentan promedios más altos en la diversidad léxica; por el contrario, textos cortos

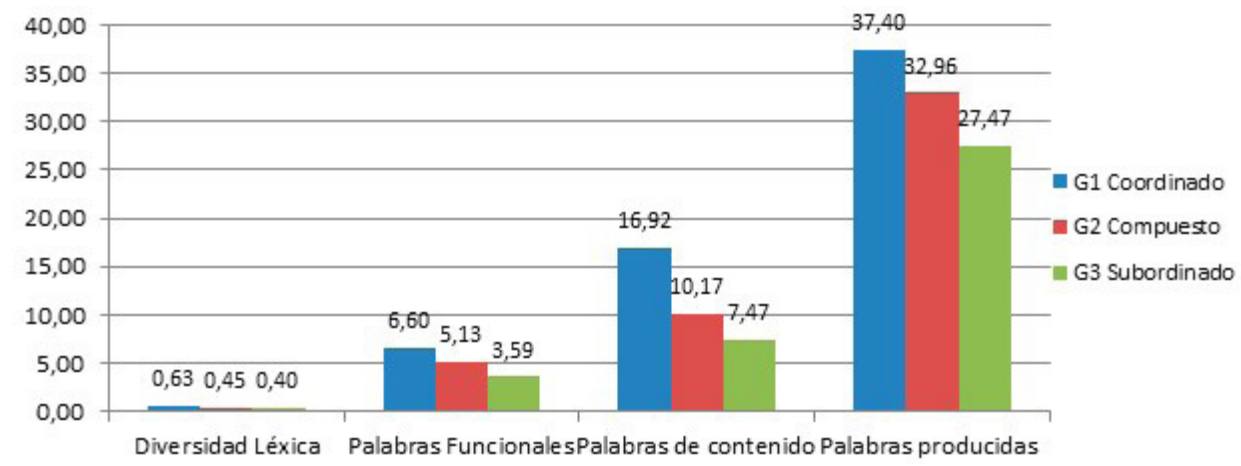

Figura 5. Diversidad léxica en inglés. Promedio de PF, PC y PP. Fuente: elaboración propia.

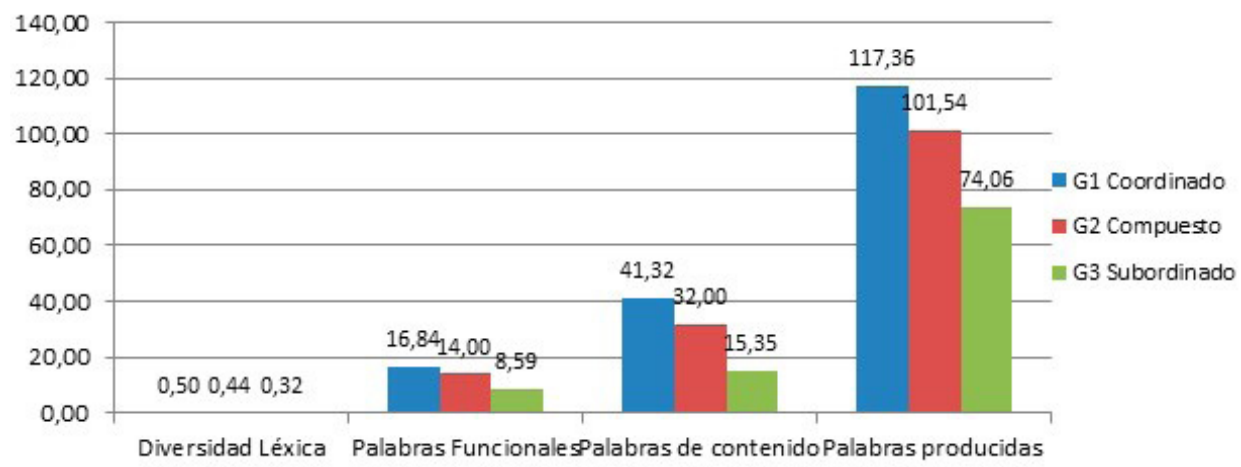

Figura 6. Diversidad léxica en español promedio de PF, promedio de PC y promedio de PP. Fuente: elaboración propia. 
como los que produjeron los sujetos de esta muestra en inglés, han mostrado tener promedios más altos en este aspecto. El promedio de PF G1 16,84, G2 14,00 y G3 8,59 y de PC G1 41,32, G2 32,00 y G3 15,35, con respecto al promedio alto de PP, evidencia una producción limitada de palabras diferentes en los textos descriptivos escritos en español. En este sentido, se aplica para la muestra de este estudio lo planteado por Rojas y Umaña (1989), el léxico, componente de la lengua, se vincula directamente con el desarrollo conceptual: en él se presenta con mayor claridad la característica de intelectuación, conectada con las posibilidades de reflexión, aprendizaje y pensamiento. Es ya común referirse a las limitaciones que respecto del uso del lenguaje se manifiestan en los escolares, pues aparte del interés que este reviste en tanto factor que ayuda a la integración del individuo a la sociedad, se relaciona estrechamente con el pensamiento. En los resultados de la diversidad léxica en español, el G2 obtuvo un promedio casi igual al de la diversidad léxica en inglés 0,44 y 0,45 respectivamente.

\section{Discusión y conclusiones}

La clasificación de los sujetos de la muestra en los diferentes tipos de bilingües permitió identificar las características de los niños en formación bilingüe de las dos instituciones educativas públicas. Los resultados de la organización cognitiva bilingüe de los sujetos se fundamentan en la propuesta de Weinreich (1953) y en la identificación de las marcas interlinguales e intralinguales en el texto descriptivo escrito en inglés. Se conformaron tres grupos: G1 coordinado, G2 compuesto y G3 subordinado. Los sujetos coordinados son el 37,87\% de la totalidad de la muestra, los compuestos el $36,38 \%$ y los subordinados el $25,75 \%$. Los sujetos en el G1 redactaron textos descriptivos en inglés, en los cuales las marcas interlinguales fueron en promedio 3,12 y las marcas intralinguales 9,60. En el G2 compuesto el promedio de las marcas interlinguales 3,83 y de las marcas intralinguales 3,33. Finalmente, en el G3 subordinado, los sujetos produjeron textos descriptivos escritos en inglés en los cuales el promedio de las marcas interlinguales 7,76 superó en gran medida el de las marcas intralinguales 1,17 .
Lo anterior permitió confirmar que el progreso del bilingüismo es un continuo que va desde el bilingüismo subordinado al bilingüismo compuesto con características relacionadas con la interlengua hasta el bilingüismo coordinado con mayores características de la intralengua (Richards, 1971). El mayor promedio de marcas interlinguales en el texto descriptivo escrito en inglés muestran que el G3 subordinado, seguido del G2 compuesto, se encuentran en el proceso de adquisición del lenguaje como el desarrollo de una competencia transicional que va dirigida hacia la $L 2$, ya que contiene características de la lengua materna L1, de la lengua extranjera L2 y de ambas mezcladas (Corder, 1967). Con respecto a las marcas intralinguales, estas muestran que el G1 coordinado se encuentra en proceso de fortalecimiento de la L1 y afianzamiento de la L2, por lo tanto, una mayor independencia entre las lenguas al momento de utilizarlas. Por último, en el grupo total de los 66 sujetos de la muestra, se dio un promedio de 4,57 en las marcas interlinguales y de 5,15 en las marcas intralinguales. Al respecto, Alexopoulou (2010) sostiene que un buen número de investigaciones han encontrado que el porcentaje de errores interlinguísticos o interlinguales en la producción de aprendices de lenguas extranjeras es claramente menor al de los errores intralingüísticos o intralinguales.

Algunos de los anteriores hallazgos estarían fundamentados en el modelo de asociación de palabras MAP (Talamás, Kroll y Dufour, 1999) que soporta la tipología bilingüe subordinada explicando que el sujeto con menor competencia lingüística en la L2 presenta más interferencias interlinguales en las respuestas en la L2 ya que posee un conocimiento restringido de esta y, por lo tanto, accede al significado de las palabras en L2 a través de la ruta lexical, es decir, a través de la forma de la palabra. De igual manera, para la tipología bilingüe compuesta el modelo de mediación conceptual MMC (Potter et al., 1984) explica que a medida que el sujeto avanza en su conocimiento de la L2, accede más al significado de las palabras de la L2 a través de la ruta conceptual y no de la léxica, lo que permite que las interferencias intralinguales en L2 aumenten porque el sujeto hace hipótesis ya desde su L2. Sin embargo, como lo observamos en los resultados 


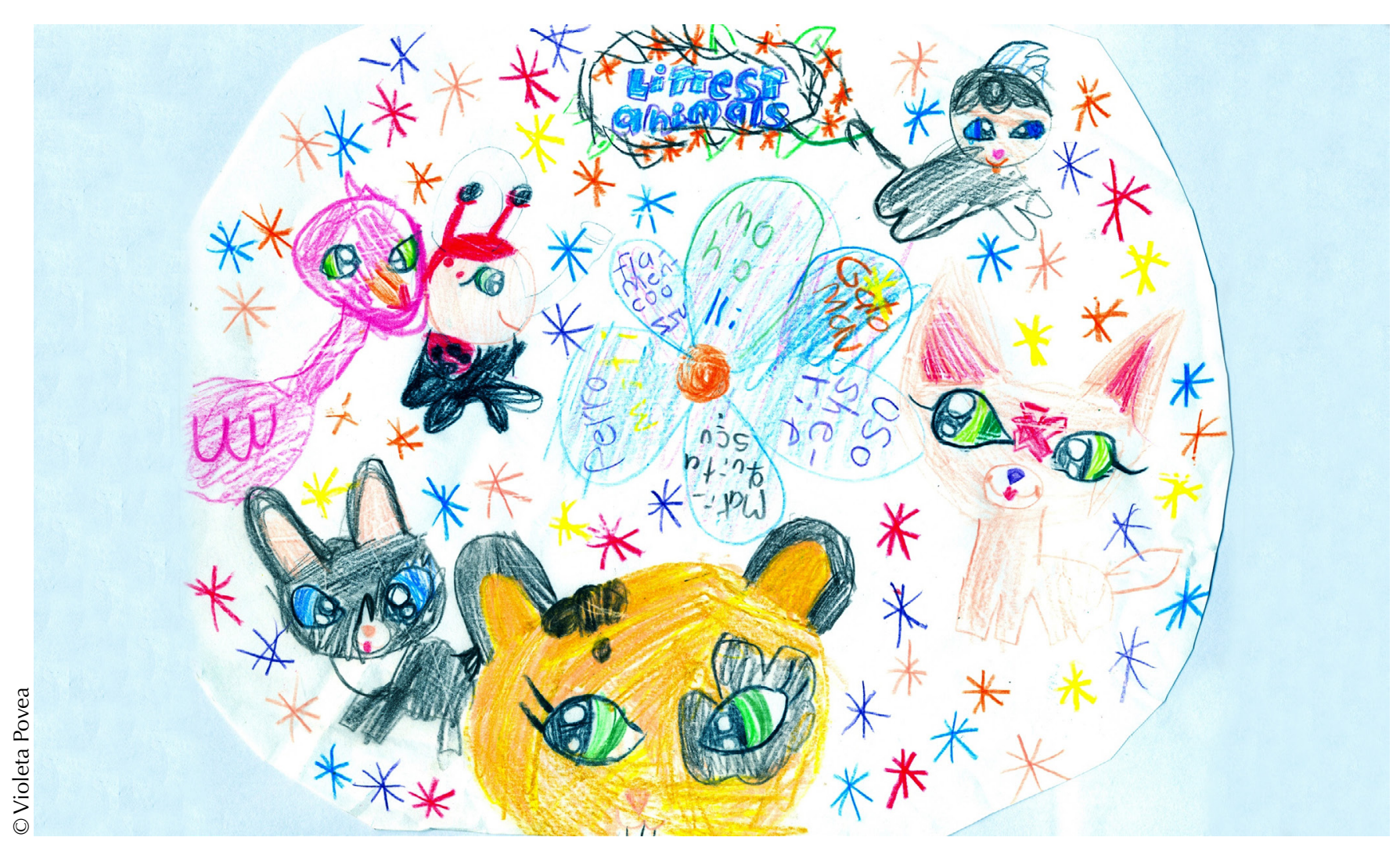

de este estudio, las interferencias interlinguales en los sujetos con tipología bilingüe compuesta siguen siendo significativas en el estadio de la interlengua.

Los sujetos de esta investigación cuya tipología bilingüe es coordinada, tuvieron los mejores desempeños en la densidad y diversidad léxica escrita en inglés y en español, seguidos por los sujetos con tipología compuesta y finalmente por sujetos con tipología subordinada. Por lo anterior, se recomienda al sistema educativo público promover la tipología de bilingüismo coordinado en los procesos de formación bilingüe en sus diferentes niveles. Esta tipología se debe desarrollar apropiadamente con los alumnos y proceder a la planificación de las actividades a llevar a cabo en el aula. Sánchez-Casas (1999) enfatiza en la necesidad de reconocer el tipo de bilingüe con el que estamos trabajando para poder garantizar una aproximación más efectiva al fenómeno del bilingüismo en el salón de clases.

Tal y como se expresó en la hipótesis de esta investigación, los sujetos en formación bilingüe de tercer grado de primaria, cuya tipología bilingüe es coordinada, alcanzan una mayor densidad y diversidad léxica en inglés y en español que aquellos sujetos que no poseen dicho rasgo cognitivo. Se esperaba que el conocimiento un poco más avanzado de la L2 por parte de los sujetos coordinados se evidenciara en el predominio en las palabras funcionales, de contenido y producidas, seguido de los sujetos compuestos y de los subordinados. Era de esperar que en esta muestra de niños en desarrollo de un bilingüismo temprano la mayoría de los sujetos se ubicara entre el G2 compuesto y G3 subordinado, ya que en su proceso la interlengua aún se mezclan las dos lenguas aun cuando existan algunas hipótesis de funcionamiento de la L2 que se elaboran desde la lengua extranjera que cada uno posee.

Los diferentes promedios alcanzados en la densidad y diversidad léxica en inglés y en español por los sujetos según su tipología bilingüe reflejaron diferencias normales en un grupo de sujetos en el cual existen factores internos y externos que marcan diferencias en el aprendizaje, entre ellos, la intensidad horaria de inglés como cátedra y como lengua vehicular, los ritmos de aprendizaje, la motivación, las estrategias de aprendizaje, entre otros. Según Justicia (1995), el desarrollo del léxico está relacionado con los contenidos escolares propios de cada nivel; aunque reconoce que no depende solo de ello, pues 
también influyen los medios de comunicación, especialmente la televisión, y las relaciones sociales con la familia y el grupo de sus iguales.

Se decidió estudiar el léxico de los niños de tercer grado de primaria, pues combina información de todos los niveles lingüísticos acerca de las palabras que forman parte del vocabulario de cada individuo en su proceso de adquisición de la L1 y la L2. De igual manera, permite observar aspectos relacionados con la ortografía, morfología, sintaxis y semántica. El desarrollo del léxico es fundamental, no solo como instrumento para promover la competencia lingüística de los niños sino como medio eficaz para el perfeccionamiento de los esquemas cognitivos infantiles, ya que el conocimiento y dominio de las palabras supone diversas formas de apropiación de los diferentes aspectos del mundo que ellas mismas designan. Un aspecto fundamental del lenguaje en los niños, subestimado en los últimos años, ha sido el desarrollo del léxico. El léxico cumple un papel relevante en el aprendizaje de la escritura (Biemiller, 2006). Las habilidades para el uso de vocabulario están asociadas con el desempeño en la escritura en los primeros años de la escuela primaria. Una de las grandes responsabilidades de la escuela es fomentar al máximo los potenciales cognitivos del niño a través de los procesos de producción del lenguaje.

\section{Referencias}

Abdelilah-Bauer. (2007). El desafío del bilingüismo. Madrid: Morata.

Auza, A.; Chávez, A. (2013). Medidas del desarrollo del lenguaje en niños mexicanos: la longitud media de emisión, diversidad y densidad léxicas en el recuento de una historia. México: Universidad Autónoma de Querétaro.

Aguado, G. (1995). El desarrollo del lenguaje de 0 a 3 años: bases para un diseño curricular en la educación infantil. Madrid: Cepe.

Alexopoulou, A. (2010). Errores intralinguales e interlinguales en la interlengua escrita de aprendientes griegos de E/LE. En P. \& F. Crémoux (eds.): Actas del XVI Congreso de la Asociación Internacional de Hispanistas: Nuevos caminos del hispanismo. CD, 2.
Baker, C. (2006). Foundations of Bilingual Education and Bilingualism ( $4^{\mathrm{a}}$ ed.). Clevedon: Multilingual Matters.

Biemiller, A. (2006). Vocabulary development and instruction: A prerequisite for school learning. En: D. K. Dickinson; S. B. Neuman (Eds.). Handbook of Early Literacy Research, Vol. 2 (pp. 4151). New York (NY): Guilford Press.

Biber, D.; Conrad, S.; Leech, G. (2002). The Longman Student Grammar of Spoken and Written English. Harlow: Longman.

Blanco, J. (2007). La lengua materna ante la globalización. Revista Colombiana de Educación Bilingüe, 1: 39-48.

Corder, P. (1967). The significance of learners errors. International Review of Applied Linguistics, 5: 161-170.

Ervin, S.; Osgood, C. (1954). Second language learning and bilingualism. Journal of Abnormal Social Psychology, suppl. 49.

Grosjean, F. (2010). Bilingual: Life and Reality. Cambridge (MA): Harvard University Press.

Hagège, C. (1996). L'enfant aux deux langues. París: Editions Jabob.

Hamers, J.; Blanc, M. (2000). Bilinguality and Bilingualism. Cambridge (UK): Cambridge University Press.

Heilmann, J. J.; Miller, J.; Nockerts, A.; Dunaway, C. (2010). Properties of the narrative retells in young school-age children. American Journal of Speech-Language Pathology, 19(2): 154-166.

Hernández, R.; Fernández, C.; Baptista, P. (2010). Metodología de la investigación. México: McGraw Hill.

Johansson, V. (2008) Lexical diversity and lexical density in speech and writing: A developmental perspective. Working Papers, 53: 61-79. Recuperado de: http://journals.lub.lu.se/index.php/ LWPL/article/view/2273/1848

Justicia, F. (1995): El desarrollo del vocabulario: diccionario de frecuencias. Granada: Universidad de Granada.

Kroll, J. F. (1993). Accessing conceptual representations for words in a second language. En: R. Schreuder; B. Weltens (eds.): The Bilingual Lexicon (pp. 54-81). Amsterdam; Philadelphia: John Benjamins. 
Lambert, W. E.; Havelka, J.; Crosby, C. (1958). The influence of language-acquisition contexts on bilingualism. The Journal of Abnormal and Social Psychology, 56(2): 239-244.

Latorre, A.; del Rincón, D.; Arnal, J. (1997). Bases metodológicas de la investigación educativa. Barcelona: Hurtado Ediciones.

Laufer, B. (1991). How much lexis is necessary for reading comprehension? En: P. J. L. Arnaud; $\mathrm{H}$. Bejoint (eds.): Vocabulary and applied linguistics (pp. 126-132). Asingstoke: Macmillan.

Lewis, E. G. (1976). Bilingualism and bilingual education: The ancient world to the Renaissance. En: J. Fishman (ed.): Bilingual Education: An International Sociological Perspective (pp. 150200). Rowley: Newbury House.

McLaughlin, B. (1984). Second-Language Acquisition in Childhood: School-Age Children. Vol. 1. Hillsdale (NJ): Lawrence Earlbaum Associates.

Murillo, M. (2009). Diversidad de vocabulario en los preescolares. Aportes para valorar su competencia léxica. Filología y lingüística, 35: 123-138.

Phillipson, R. (1992). Linguistic Imperialism. Oxford: Oxford University Press.

Potter, M. C.; So, K. F.; Von Eckardt, B.; Feldman, L. (1984). Lexical and conceptual representation in beginning and proficient bilinguals. Journal of Verbal Learning and Verbal Behavior, 23(1): 23-38.

Richards, J. (1971). Error analysis and second language strategies. Language Sciences, 17: 12-22.

Rojas, M. (2005). Una responsabilidad escolar olvidada: el desarrollo del componente léxico. Revista Educación, 29(1): 31-44.
Rojas, M.; Umaña, R. (1989). Sobre la construcción léxica. En: Káñina. Revista de Artes y Letras, 13(1-2).

Sánchez-Casas, R. M. (1999). El léxico del hablante bilingüe. En: M. De Vega; F. Cuetos (eds.): Psicolingüística del español. Valladolid: Trotta.

Signoret, A.; Da Silva, H. (2011). L'Acquisition $d^{\prime}$ une langue seconde ( $1^{\text {a }}$ ed. en francés). México: Universidad Nacional Autónoma de México (UNAM).

Snow, C. E. (1999). Age differences in second language: Research findings and folk psychology. En: N. Baley (Ed.). Second Language Acquisition Studies (pp. 141-149). Rowley (MAss.): Newbury House.

Swanson, L. A.; Fey, M.; Mills, C. E.; Hood, L. S. (2005). Use of narrative-based language intervention with children who have specific language impairment. American Journal of Speech-Language Pathology, 14: 131-141.

Talamás, A.; Kroll, J. F.; Dufour, R. (1999). Form related errors in second language learning: A preliminary stage in the acquisition of L2 vocabulary. Bilinguism: Language and Cognition, 2: 45-58.

Uccelli, P.; Páez, M. (2007) Narrative and vocabulary development of bilingual children from kindergarten to first grade. Developmental changes and associations among English and Spanish. Language, Speech, and Hearing Services in Schools, 38: 225- 236.

Weinreich, U. (1953). Languages in Contact: Findings and Problems. New York: Publications of the Linguistic Circle of New York. 